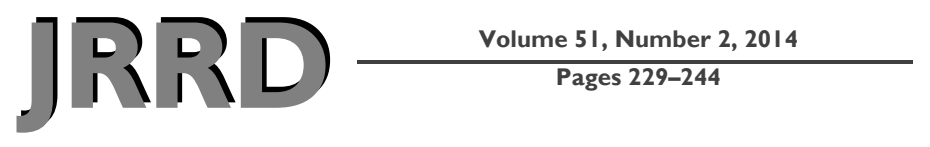

\title{
Sensor-based hip control with hybrid neuroprosthesis for walking in paraplegia
}

\author{
Curtis S. To, PhD; ${ }^{1}$ Rudi Kobetic, MS; ${ }^{*}$ Thomas C. Bulea, PhD; ${ }^{2}$ Musa L. Audu, PhD; ${ }^{1-2}$ John R. Schnellenberger, \\ MS; ${ }^{1}$ Gilles Pinault, $\mathbf{M D}{ }^{1}{ }^{1}$ Ronald J. Triolo, $\mathbf{P h D}^{1-3}$ \\ ${ }^{1}$ Louis Stokes Cleveland Department of Veterans Affairs Medical Center, Cleveland, OH; Departments of ${ }^{2}$ Biomedical \\ Engineering and ${ }^{3}$ Orthopaedics, Case Western Reserve University, Cleveland, $\mathrm{OH}$
}

\begin{abstract}
The objectives of this study were to test whether a hybrid neuroprosthesis (HNP) with an exoskeletal variableconstraint hip mechanism (VCHM) combined with a functional neuromuscular stimulation (FNS) controller can maintain upright posture with less upper-limb support and improve gait speed as compared with walking with either an isocentric reciprocating gait orthosis (IRGO) or FNS only. The results show that walking with the HNP significantly reduced forward lean in FNS-only walking and the maximum upper-limb forces by $42 \%$ and $19 \%$ as compared with the IRGO and FNS-only gait, respectively. Walking speed increased significantly with VCHM as compared with 1:1 reciprocal coupling and by $15 \%$ when using the sensor-based FNS controller as compared with HNP with fixed baseline stimulation without the controller active.
\end{abstract}

Key words: exoskeleton, functional neuromuscular stimulation, gait, hybrid neuroprosthesis, reciprocating gait orthosis, sensor-based hip control, spinal cord injury, trunk support, walking, walking in paraplegia.

\section{INTRODUCTION}

Trunk-hip-knee-ankle-foot orthoses (THKAFOs) for restoring gait after paralysis from spinal cord injury (SCI) provide upper-body postural support by means of a thoracic jacket coupled to the hips. For example, the hip guidance orthosis [1] constrains all hip joint motion to the sagittal plane, thus providing coronal trunk support. However, the range of hip flexion is limited with mechanical stops to prevent excessive forward trunk lean [2]. The reciprocating gait orthosis (RGO) [3] also constrains trunk and hip motion in the coronal plane and incorporates a mechanism that reciprocally couples hip flexion with contralateral hip extension. During stance, the RGO provides trunk support and reciprocal stepping initiated by contralateral hip extension. However, because sagittal hip motion is linked to trunk motion through a rigid corset, contralateral hip extension requires posterior trunk motion by means of the upper limbs, which is counterintuitive for stepping forward and may be confusing for the user. Furthermore, reciprocal coupling between the hips is fixed at a 1:1 hip flexion and extension ratio, limiting step length [4]. These compromises, however, have not discouraged continued development to improve the functional performance of the RGO since reciprocal hip

Abbreviations: FNS = functional neuromuscular stimulation, $\mathrm{FSR}=$ force sensitive resistor, HNP $=$ hybrid neuroprosthesis, IRGO = isocentric reciprocating gait orthosis, $\mathrm{PW}=$ pulse width, $\mathrm{RGO}=$ reciprocating gait orthosis, $\mathrm{SCI}=$ spinal cord injury, SCKM = stance-control knee mechanism, THKAFO = trunk-hip-knee-ankle-foot orthosis, UECU = universal external control unit, VCHM = variable-constraint hip mechanism.

*Address all correspondence to Rudi Kobetic, MS; Louis Stokes Cleveland VA Medical Center, 10701 East Blvd, 151A, Cleveland, OH 44106; 216-791-3800, ext 4694; fax: 216-231-3433. Email: rudolf.kobetic@va.gov http://dx.doi.org/10.1682/JRRD.2012.10.0190 
coupling has been shown to maintain trunk posture [4-5], which may have the effect of reducing energy costs [6-7].

Initial improvements to the RGO ranged from simplifying the hip reciprocating mechanism using Bowden cables $[3,8]$ for torque transfer to a simple pivoting bar and tie rod design known as the isocentric RGO (IRGO) [9]. A modified hip mechanism in the $\mathrm{R}^{2} \mathrm{GO}$ [10] facilitates the pelvic rotation characteristic of normal gait by coupling hip flexion with ipsilateral external hip rotation and hip extension with ipsilateral internal hip rotation in addition to reciprocal coupling between the hips in the sagittal plane. The $\mathrm{R}^{2} \mathrm{GO}$ reduced vertical displacement of the center of mass by $8 \mathrm{~cm}$ relative to that of an RGO in an individual with SCI and resulted in a more efficient pattern of horizontal pelvic rotation [10]. Other innovations involved the application of external power assistance to the RGO including direct current motors [11-13] and pneumatic artificial muscles [14] to help drive the reciprocal motion of the RGO. Such power-assisted RGOs decreased effort by users with SCI relative to walking with a standard RGO and have been shown to reduce the lateral and vertical compensatory motions involved in standard RGO gait $[11,15]$. However, improvements to the standard RGO resulted in minimal increase in walking speed primarily with increased cadence since the constraints imposed on the sagittal kinematics by the $1: 1$ reciprocal hip coupling limiting step length were not addressed [16]. A promising new approach involves combining functional neuromuscular stimulation (FNS) with controllable exoskeletons to provide a more efficient power for ambulation with potential for the physiological benefits of active muscle contraction [17].

An RGO configured for a 2:1 reciprocal hip flexion and extension coupling directed toward more physiological kinematics both increased stride length and decreased energy cost, with appreciable increase in gait speed when hip flexion was assisted by FNS [18]. However, the postural support provided by RGOs are intermittent and only beneficial during the double support phases of gait and actually hinder lower-limb kinematics during the rest of the gait cycle [5]. Thus, a hydraulic variable-constraint hip mechanism (VCHM) was developed [19] to actively modulate the reciprocal coupling of the hips (Figure 1). The VCHM was designed to reciprocally couple the hips or independently free or lock a hip against motion through sensor signal feedback control. Hip reciprocation would only be active during instances of trunk instability as dictated by sensor feedback; otherwise, the hip would be

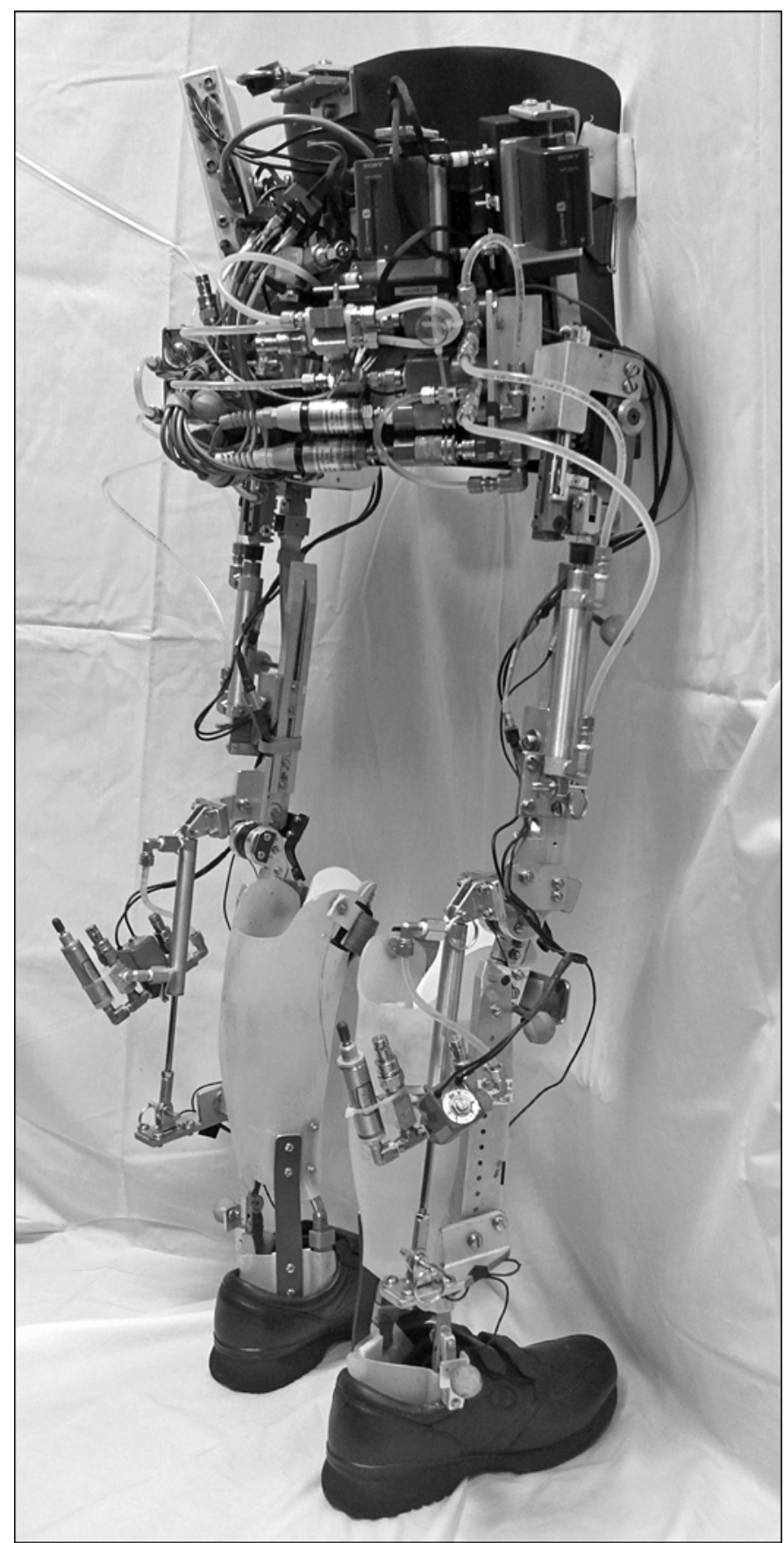

Figure 1.

Exoskeleton portion of hybrid neuroprosthesis consisting of hydraulic variable-constraint hip mechanism, stance-control knee mechanisms, and locked ankle-foot orthoses.

freed to allow for variable step lengths. The VCHM was envisioned to work in conjunction with FNS, which would provide the power to actively rotate the hip joints 
through the stimulated contraction of the flexor and extensor muscles.

In this study, the VCHM was incorporated into a hybrid neuroprosthesis (HNP) that utilized FNS to power lower-limb movements and a THKAFO to provide support. The overall objective was to determine the effects of the VCHM combined with the sensor-based control of FNS on trunk orientation, upper-limb forces on a walking aid, and step length relative to existing gait interventions for functional compensation of walking in paraplegia. The following hypotheses were tested as part of this study: (1) the VCHM can maintain upright walking posture comparable with the IRGO; (2) walking with an HNP incorporating the VCHM requires less upper-limb support than IRGO-only and FNS-only systems; (3) the VCHM allows the FNS to improve hip flexion during swing, with step length comparable with FNS-only gait; and (4) a rule-based feedback FNS controller in combination with the VCHM improves hip dynamics and gait speed.

\section{METHODS}

\section{Test Cases}

An HNP consisting of an exoskeleton and an implanted FNS system was implemented and evaluated with a sensor-based controller for walking after paralysis from SCI. The exoskeleton weighing $22.2 \mathrm{~kg}$ was a modified IRGO where the reciprocating bar was replaced with the VCHM and the drop-lock knee joints were replaced with a pair of prototype hydraulic stance-control knee mechanisms (SCKMs) [19-20]. The SCKM was necessary to lock the knee in extension against collapse during stance while allowing for free motion during FNS-driven swing (Figure 1). Multiple control and experimental cases were examined to quantify the rule-based feedback (sensorbased) control of VCHM and FNS (Table 1). The results were compared with control cases representing conventional interventions: (1) walking with an FNS only with preprogrammed muscle stimulation patterns (open-loop FNS) and (2) walking with an IRGO only with reciprocally coupled hips and knees and ankles locked at full extension and neutral, respectively. A system combining the VCHM, bilateral SCKMs, and open-loop FNS (i.e., $\left.\mathrm{HNP}_{\text {open }}\right)$ was compared with walking with the HNP to quantify the effect of the rule-based feedback FNS controller. The effect of the hip control mechanism was quantified by comparing the $\mathrm{HNP}_{\text {open }}$ with a walking system that
Table 1.

Assistive gait device configurations.

\begin{tabular}{lllll}
\hline \multirow{2}{*}{$\begin{array}{c}\text { Assistive } \\
\text { Device }\end{array}$} & \multicolumn{4}{c}{ Joint Constraint } \\
\cline { 2 - 5 } IRGO Only & IRGO & Knee & Ankle & FNS \\
FNS Only & None & None & Locked & None \\
HNP $_{\text {IRGO }}$ & IRGO & SCKM & Locked & Open-Loop \\
HNP $_{\text {open }}$ & VCHM & SCKM & Locked & Open-Loop \\
HNP & VCHM & SCKM & Locked & Rule-Based \\
& & & & Feedback \\
\hline
\end{tabular}

$\overline{\mathrm{AFO}}=$ ankle-foot orthosis, $\mathrm{FNS}=$ functional neuromuscular stimulation, $\mathrm{HNP}=$ hybrid neuroprosthesis, IRGO = isocentric reciprocating gait orthosis, SCKM = stance-control knee mechanism, VCHM = variable-constraint hip mechanism.

combined bilateral SCKMs and open-loop FNS with the standard reciprocating bar of the IRGO (i.e., $\mathrm{HNP}_{\text {IRGO) }}$ ).

\section{Rule-Based Feedback Control of Variable-Constraint Hip Mechanism}

The VCHM was designed as a closed hydraulic system to couple, free, or lock the hips [19]. The mechanism provides stance hip support by constraining the hip against flexion while allowing it to extend by means of hip extensor stimulation during contralateral swing. By freeing the hip from flexion constraint, the leg is free to swing, allowing variable step length with the FNS-driven hip musculature. The upright trunk posture is maintained by coupling of the hips, which prevents bilateral hip flexion in order to minimize the use of upper limbs for support.

A modified finite state machine [21] was designed to control the VCHM during standing and walking (Figure 2).

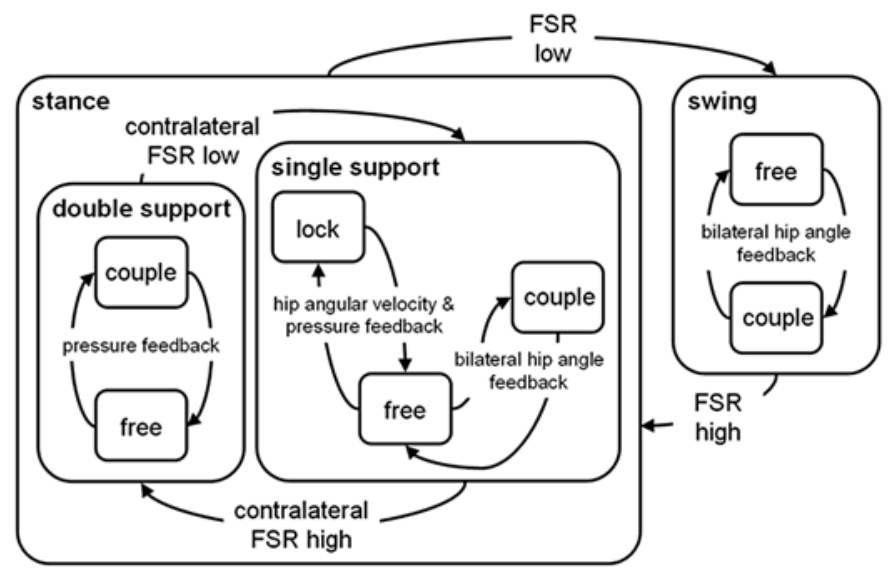

Figure 2.

Finite state machine diagram for variable-constraint hip mechanism controller. FSR = force sensitive resistor. 
This VCHM controller used feedback signals from (1) footground contact from force sensitive resistors (FSRs) (B \& L Engineering; Santa Ana, California) embedded under the insoles of the shoes, (2) hip angles from potentiometers (Alps Electric Co; Tokyo, Japan), (3) hip angular velocities from numerical differentiation of hip angle signals, and (4) cylinder pressures from digital pressure transducers (Gems Sensors \& Controls; Plainville, Connecticut) mounted at each port of the two hydraulic cylinders of the VCHM. All the sensors were permanently attached to the exoskeleton.

Operation of the VCHM required real-time detection of double support, single support, and swing phases of the gait cycle (Figure 2). The rules to transition the VCHM among coupled, freed, and locked states were based on an event detector that discriminated among the gait phases using foot-ground contact signals derived from the FSRs. A high signal from either heel or forefoot FSR, designated by two set thresholds, indicated that the corresponding foot was in contact with the ground, whereas the foot was designated as off the ground only if both the heel and forefoot FSR signals were below a specified threshold.

Hip joint trajectories were defined by FNS and controlled by the real-time, rule-based controller to maintain erect posture and improve step length. Because the rigid thoracic jacket couples pelvis to trunk, any hip flexion results in forward lean of the trunk. During the double support phases of gait, the hips were reciprocally coupled to prevent bilateral hip flexion. However, the feedback from the VCHM cylinder pressures allowed for hip extension to facilitate posture correction by the user. During single support, the stance hip was freed to extend under FNS control. Any stance hip flexion (i.e., forward trunk tilt) was prevented by the unidirectional locking of the hip joint against further hip flexion based on ipsilateral hip angular velocity and VCHM pressure feedback. Failure of the stance hip to extend with stimulation would result in bilateral hip flexion, which initiated reciprocal coupling such that the contralateral FNS-controlled hip flexion assisted extension of the stance hip. Note that locking the stance hip against flexion was chosen to be the initial response to insufficient extension over reciprocally coupling the hips, which might otherwise impede swing hip flexion and hence reduce step length. Whether through locking or reciprocal coupling, the stance hip flexion was impeded to minimize anterior trunk tilt.
The hip was freed throughout the swing phase, allowing the VCHM to accommodate any step length achievable by FNS. However, if the contralateral stance hip was unable to extend efficiently, the hips were reciprocally coupled during swing. Thus, this control scheme allowed free hip flexion during swing for increasing the step length, provided that stimulation of hip flexors could drive the contralateral limb into extension. The controller parameters were defined through preliminary bench [19$20]$ and nondisabled testing.

\section{Rule-Based Feedback Control of Functional Neuromuscular Stimulation}

Real-time modulation of FNS was synchronized with operation of the exoskeleton through sensor feedback. The goal was to achieve desired end points in the joint trajectories during gait and deactivate target muscles once these end points had been met. In this study, only stimulation to hip extensors was modulated by the rulebased feedback controller to maintain posture and power for forward progression.

The FNS controller was implemented to maintain functional hip extension motion throughout single stance. Stimulation parameters within the safe limits of stimulation with intramuscular electrodes were used with constant current stimulus pulses of $20 \mathrm{~mA}$ in amplitude and pulse width (PW) modulated between 0 and $250 \mu$ s [22]. Muscle fiber recruitment was modulated with stimulus PW [23], while additional force was generated by increased frequency [24]. Due to software limitations, stimulus frequency could only be switched between 16.67 and $33.33 \mathrm{~Hz}$. The lower frequency was used mostly to minimize muscle fatigue [25]. Stimulation to the hip extensor muscles was modulated relative to a baseline pattern (i.e., the preprogrammed stimulation for open-loop FNS-only walking) according to the following three rules.

\section{Rule 1}

If the hips are coupled by the VCHM during single stance, then the stimulation $P W$ and frequency of the stance limb hip extensors are maximally increased. Single stance occurred when the ipsilateral SCKM was locked and contralateral SCKM was unlocked. Reciprocal coupling during single stance occurred when both hips were flexed such that contralateral hip flexion assisted ipsilateral hip extension. To maximize hip extension and minimize the impediment to contralateral flexion, stimulation to the ipsilateral hip extensors was increased. 
Rule 2

If the hip is locked against flexion during single stance by the VCHM, then the stimulation PW and frequency of the stance limb hip extensors are maximally increased. This rule was designed to ensure continuous hip extension during single stance to maintain forward propulsion and prevent anterior trunk tilt.

\section{Rule 3}

If the stance hip is fully extended, then the stimulation to the stance hip extensors is deactivated. Stance was defined by a locked SCKM. The hip is fully extended once the hip angle crosses below a first threshold $\left(-8^{\circ}\right.$, hip extension is negative) and is considered fully extended as long as it remains greater than a second hip angle threshold $\left(0^{\circ}\right)$. The difference between the first and second hip angle thresholds is the acceptable compliance of the VCHM while it is constraining the hip against flexion, which was determined in bench testing to be approximately $8^{\circ}$ at a locking torque of $35 \mathrm{Nm}$ [20]. Rule 3 took precedence over rule 2 . Thus, once the stance hip was fully extended, stimulation to hip extensors was deactivated. Any stance hip flexion would then be prevented by the VCHM, locking the hip against flexion.

\section{Controller Implementation}

The VCHM, SCKM [19,26-27], and FNS controllers were implemented in MATLAB $^{\circledR}$, Simulink ${ }^{\circledR}$, and xPC Target (The MathWorks Inc; Natick, Massachusetts). A target computer ran the controller application in real time and processed analog and digital input and output (data acquisition) (National Instruments; Austin, Texas) for feedback signal acquisition and control output to the hip and knee mechanisms of the exoskeleton. The exoskeleton was tethered to the target computer via $20 \mathrm{~m}$ of shielded multiconductor cabling. The sampling frequency of the controllers was set at $200 \mathrm{~Hz}$. The VCHM potentiometer signals were low-pass filtered (5th-order Butterworth) at a cutoff frequency of $10 \mathrm{~Hz}$, while the pressure and FSR signals were low-pass filtered (7th-order Butterworth) at a cutoff frequency of $20 \mathrm{~Hz}$. A custom MATLAB ${ }^{\circledR}$ graphical user interface on a host computer was used for the building, sensor zeroing, and tuning of the controllers on the target computer. Biphasic charge-balanced asymmetric pulses for each channel of muscle stimulation were output by a custom computer-controlled stimulator called the universal external control unit (UECU) [28], which was attached to the exoskeleton. A $20 \mathrm{~m}, 550 \mathrm{MHz}$ patch cable was used to connect the UECU to the target computer. PW and frequency parameters were updated on the UECU by the target computer every $30 \mathrm{~ms}$.

\section{Evaluation}

A within-subject experimental design was used to test hypotheses on the effect of different walking system configurations. While practice and carryover effects could be major disadvantages of this study design, precautions were taken to minimize them in this study. Because the main purpose of this study was to test the feasibility of the new hip control strategy, a single subject with implanted electrodes was sufficient for testing. While this study has limited generalizability, it can be useful for developing and refining new HNPs for walking in paraplegia. It will help in defining the outcome measures and the effect size that produce clinically meaningful improvements for future, larger-scale studies.

A male with a thoracic level 9, American Spinal Injury Association A class SCI consented to participate in this study approved by the local institutional review board. He was $64 \mathrm{yr}$ old, $70 \mathrm{~kg}, 158 \mathrm{~cm}$ tall, and an experienced FNS user. He received a multichannel FNS-only system over $25 \mathrm{yr}$ prior to enrollment and used it to exercise approximately $1 \mathrm{~h}$ per day and walk approximately five times per week for about $200 \mathrm{~m}$ per session with standby assistance [29].

He had 22 percutaneous intramuscular electrodes implanted to control his hip flexors (iliopsoas), hip and knee flexors (sartorius, gracilis, tensor fasciae latae), hip extensors (hamstrings, gluteus maximus, posterior portion of adductor magnus), knee extensors (quadriceps without rectus femoris), ankle dorsiflexors and evertors (tibialis anterior and peroneus longus), and ankle plantar flexors (gastrocnemius). Two electrodes were implanted for redundancy to control quadriceps and a single electrode recruited both tibialis anterior and peroneus longus to achieve balanced ankle dorsiflexion. In addition, surface electrodes were used to activate the trunk extensors. Preprogrammed patterns of stimulation for FNS-only walking (Figure 3) were generated according to a rule-based algorithm for synthesis of paraplegic gait [30] and were used as a baseline for the FNS controller. Stimulation patterns for sitting and standing were adjusted heuristically based on user feedback. Stimulated responses between legs varied somewhat due to electrode position, muscle fiber type, and joint passive properties. For all test cases involving FNS, Figure 3 shows the baseline stimulus patterns used. The light and dark bands indicate stimulation frequencies of 16.67 and $33.33 \mathrm{~Hz}$, respectively. 


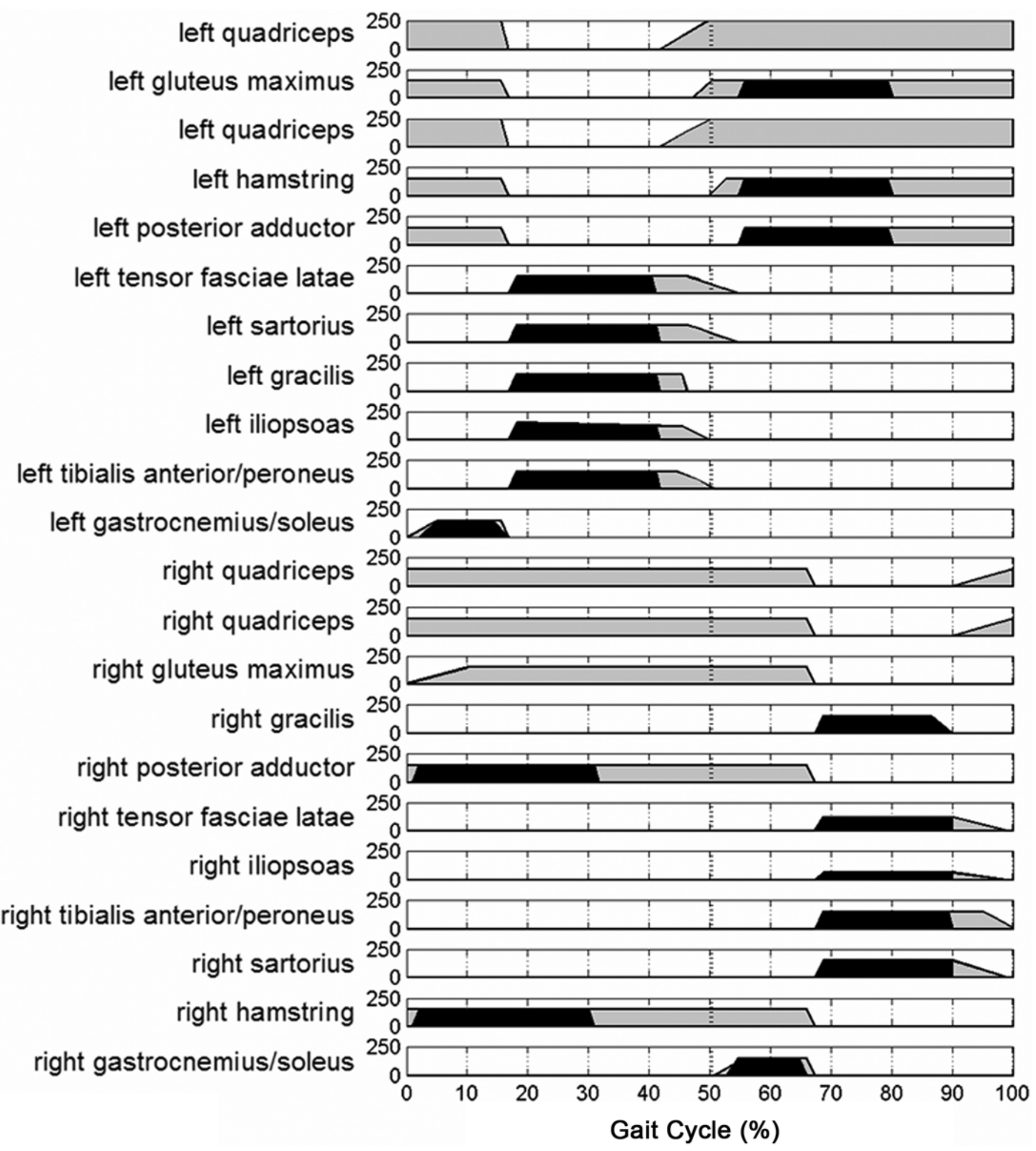

Figure 3.

Baseline stimulation pulse width pattern (microseconds) for all muscles targeted for functional neuromuscular stimulation during right stride. Light and dark regions indicate stimulus frequency of 16.67 and $33.33 \mathrm{~Hz}$, respectively. 
Normally, the low-stimulation frequency producing fused muscle contraction would be preferable to minimize muscle fatigue. Thus, short bursts of higher-frequency stimulation were used to attain immediately strong and rapid contractions that were followed by lower-frequency stimulation to maintain the responses. For example, highfrequency stimulation was used for the hip and knee flexors and ankle dorsiflexors to accelerate the leg into swing. During terminal swing, the frequency was reduced in preparation for heel strike. High-frequency stimulation was also delivered to the hip extensors during loading to provide extra extension moment for moving the body forward, while low-frequency stimulation was used during mid and terminal stance to maintain hip extension. Lowfrequency stimulation was, therefore, the default state and applied for longer periods of time during the gait cycle than high-frequency stimulation.

To don each exoskeleton configuration, the participant transferred from his wheelchair into a chair on which the exoskeleton was placed with the thigh uprights abducted and the knees flexed. The exoskeleton was fastened to the user via straps across the lower torso, pelvis, and just below the knee, and shoes were worn over bilateral fixed ankle-foot orthoses. Hip adduction and abduction were locked at neutral by a spring-loaded drop lock.

The vertical components of the forces applied to the walker by the upper limbs were measured with load cells (AMTI Inc; Watertown, Massachusetts) attached to each handle (Figure 4) and low-pass filtered (7th-order Butterworth) at a cutoff frequency of $20 \mathrm{~Hz}$. For all experimental cases, upper-limb forces were normalized to the participant's body weight in order to determine the reduction of the upper-limb effort regardless of the weight of the device (the IRGO and SCKM weighed $11 \mathrm{~kg}$ while the VCHM and SCKM weighed $22 \mathrm{~kg}$ ). The resistive hip torque by the exoskeleton during walking was calculated from the VCHM hydraulic pressure data and transmission geometry.

A 16-camera Vicon MX40 (Vicon Inc; Oxford, United Kingdom) motion analysis system (sampling at $100 \mathrm{~Hz}$ ) recorded the three-dimensional coordinates of reflective markers placed on the exoskeleton and/or bony landmarks of the body [31]. Trunk and joint kinematics, gait speed, cadence, and step length were derived from the marker coordinates offline. The target computer used for control and the Vicon system computer used for performance analysis were synchronized by a digital pulse sent from the target computer to the Vicon system computer at the beginning of data acquisition.

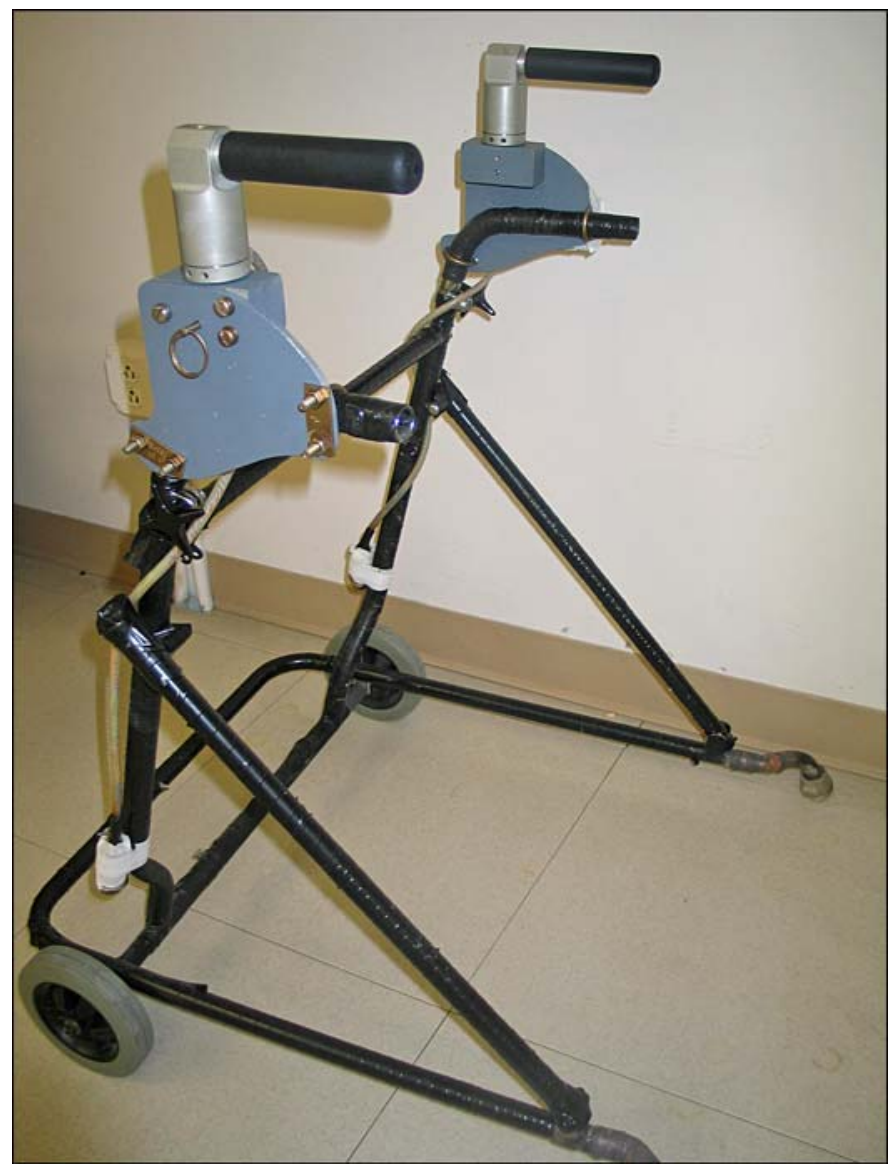

Figure 4.

Load cell mounting on walker for measuring upper-limb forces.

For each test case, the participant walked along an $8 \mathrm{~m}$ walkway at his preferred speed for six trials. IRGO-only, FNS-only, and $\mathrm{HNP}_{\mathrm{RGO}}$ walking systems were tested on separate days with 5 min rests between trials. The $\mathrm{HNP}_{\text {open }}$ and the HNP with FNS controller were tested on two separate days in a random order with the subject blinded to the configuration used. For FNS-only walking, the free cycling preprogrammed pattern of stimulation was initiated and stopped by a finger switch and adjusted to the subject's comfortable speed. For all other test cases involving FNS, each step was triggered manually by the user via a finger switch at his preferred rate. Under all conditions, a spotter walked alongside the participant for safety. Data were analyzed and averaged with respect to percentage gait cycle with \pm 1 standard deviation. The gait cycle was resolved from heel strikes that were determined from the vertical minima of the calcaneus marker coordinates in the vertical axis of the Vicon work volume. Data from each leg were considered to be independent and analyzed separately. The 
mean and average maximum gait kinematics were determined from approximately 20 strides for each test case. Analysis of variance with 95 percent confidence $(p<0.05)$ determined statistical significance between experimental and control cases. Figure 5 shows the participant walking with three configurations of the assistive systems: IRGO only, FNS only, and HNP with rule-based feedback control.

\section{RESULTS}

\section{Variable-Constraint Hip Mechanism Effect on Trunk Orientation}

Figure 6 summarizes the mean and average maximum trunk orientation for all test cases. There was on average $10^{\circ}(p<0.001)$ more anterior trunk lean with the $\mathrm{HNP}_{\text {open }}$ than walking with the IRGO only or with $\mathrm{HNP}_{\text {IRGO }}(p<0.001)$. This additional amount of forward trunk tilt may be because of a combination of VCHM compliance and any forward trunk tilt during sensor zero calibration. In spite of this modest amount of forward trunk tilt, the VCHM was providing significant support resisting up to $12 \mathrm{Nm}$ of hip flexion torque to maintain posture during walking. The mean and average maximum forward trunk lean of the $\mathrm{HNP}_{\text {open }}$ were, respectively, 36 percent $(p<0.001)$ and 22 percent $(p<0.001)$ less than during FNS-only walking. No additional improvements in posture were noted with sensor-based FNS control. These results indicate that the VCHM maintained

\section{IRGO-only}

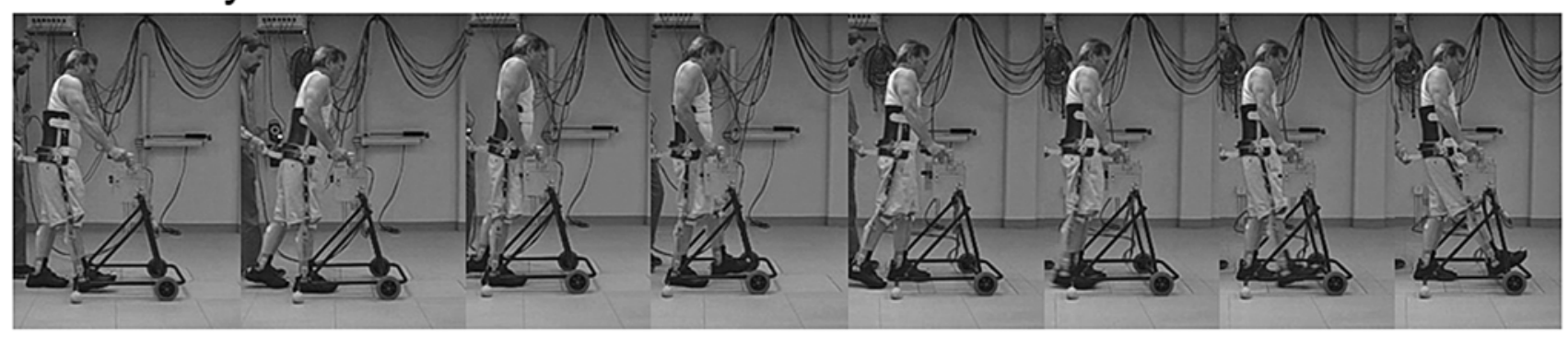

\section{FNS-only}

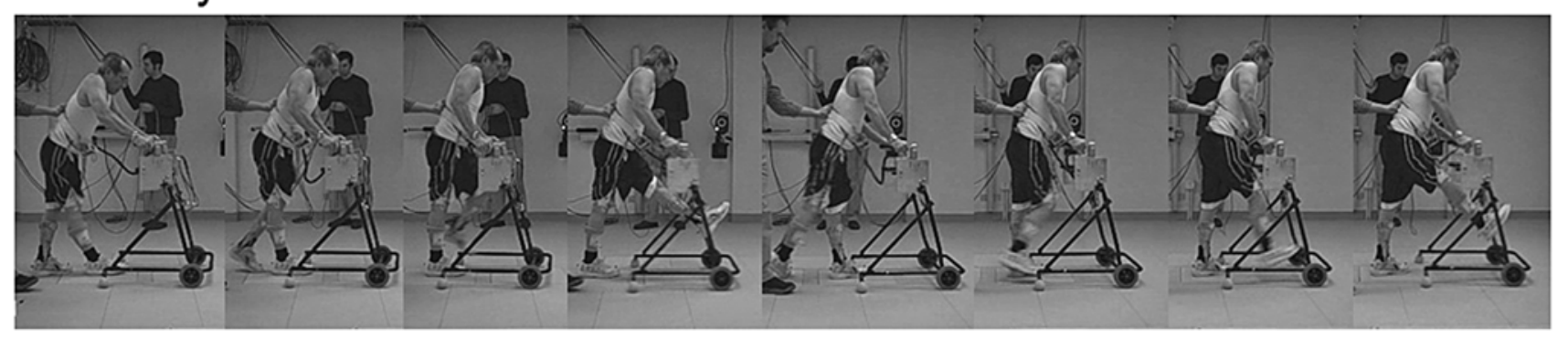

\section{HNP}

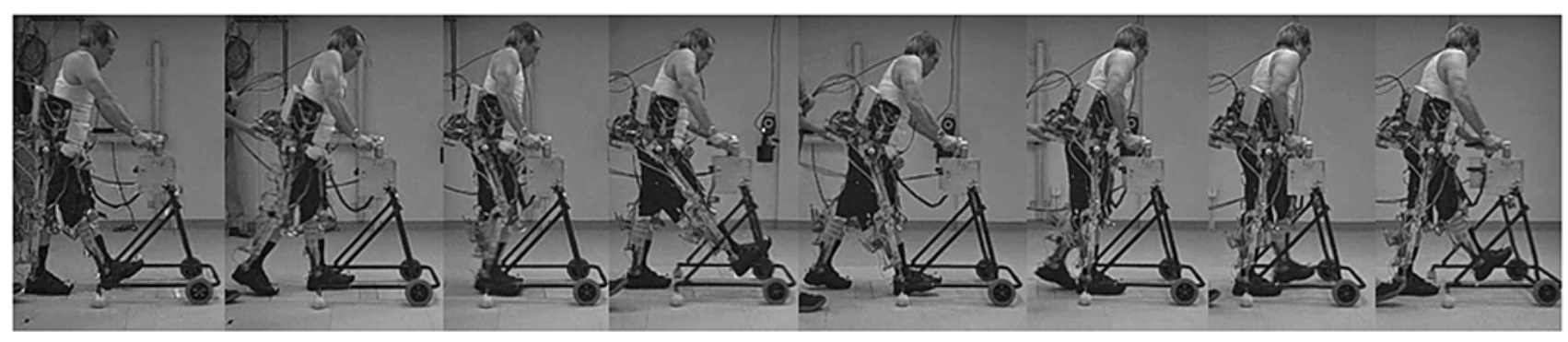

Figure 5.

Typical gait cycle is shown with isocentric reciprocating gait orthosis (IRGO) only, functional neuromuscular stimulation (FNS) only, and hybrid neuroprosthesis (HNP) with rule-based feedback control. 
trunk posture significantly better than the actions of hip and trunk stimulation assisted by the upper limbs in FNSonly gait and not as well as IRGO.

\section{Variable-Constraint Hip Mechanism Effect on Upper-} Limb Forces

The mean and average maximum upper-limb forces applied to the walker for the $\mathrm{HNP}_{\text {open }}$ were, respectively, 8 percent $(p=0.01)$ and 42 percent $(p<0.001)$ less than during IRGO-only walking and, respectively, 28 percent

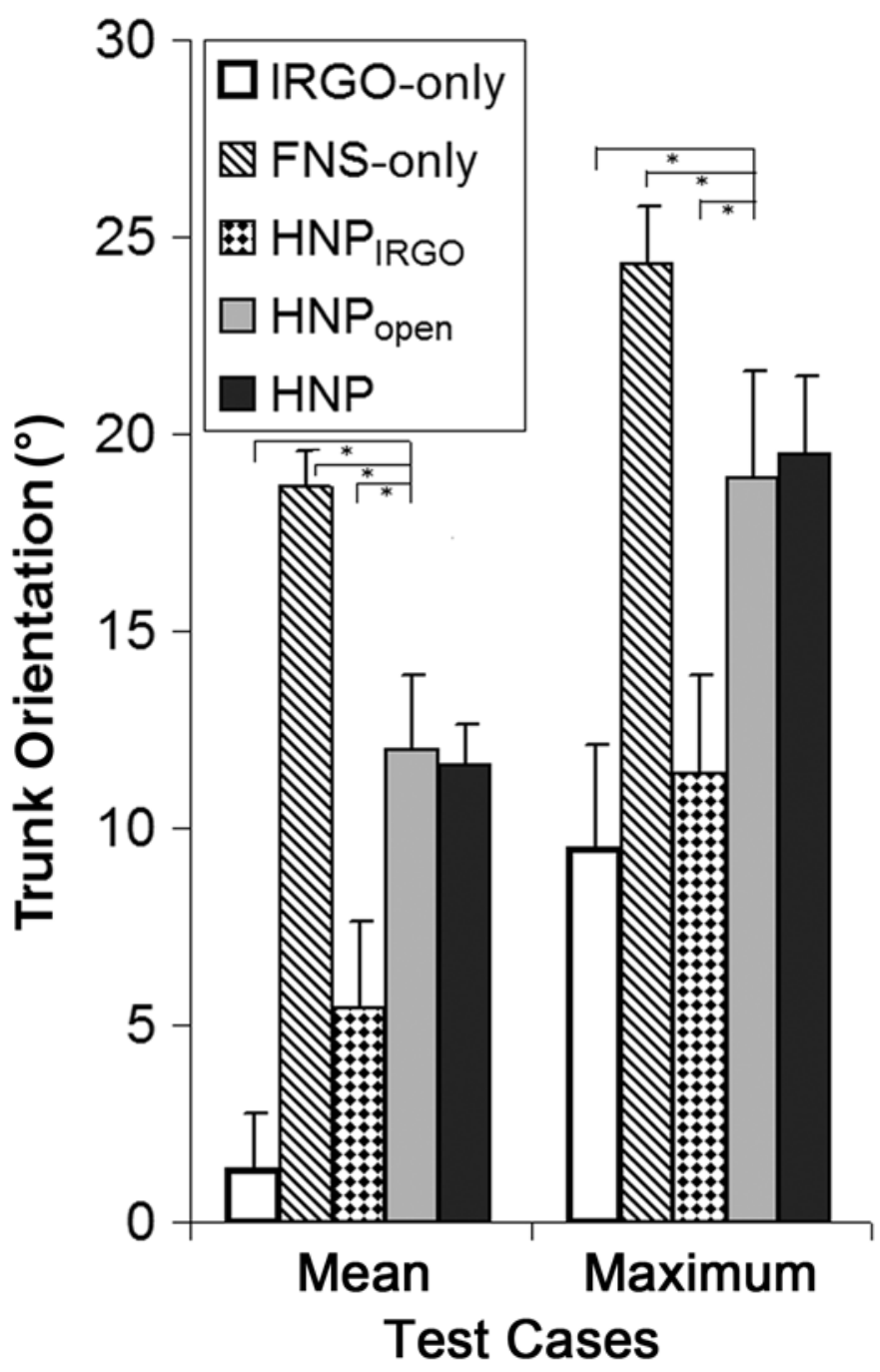

Figure 6.

Mean and average maximum forward trunk orientation (+1 standard deviation) for all test cases. *Statistical significances between system configurations. FNS = functional neuromuscular stimulation, HNP = hybrid neuroprosthesis, IRGO = isocentric recriprocating gait orthosis. $(p<0.001)$ and 19 percent $(p<0.001)$ less than for the FNS-only walking (Figure 7$)$. The mean and average maximum upper-limb forces were 9 percent $(p=0.04)$ higher and 12 percent $(p=0.03)$ lower when using the VCHM instead of the IRGO in the hybrid configuration. These results show that the $\mathrm{HNP}_{\text {open }}$ decreased the upper-limb loads relative to FNS-only gait and contributed to reducing the higher loads observed with the use of

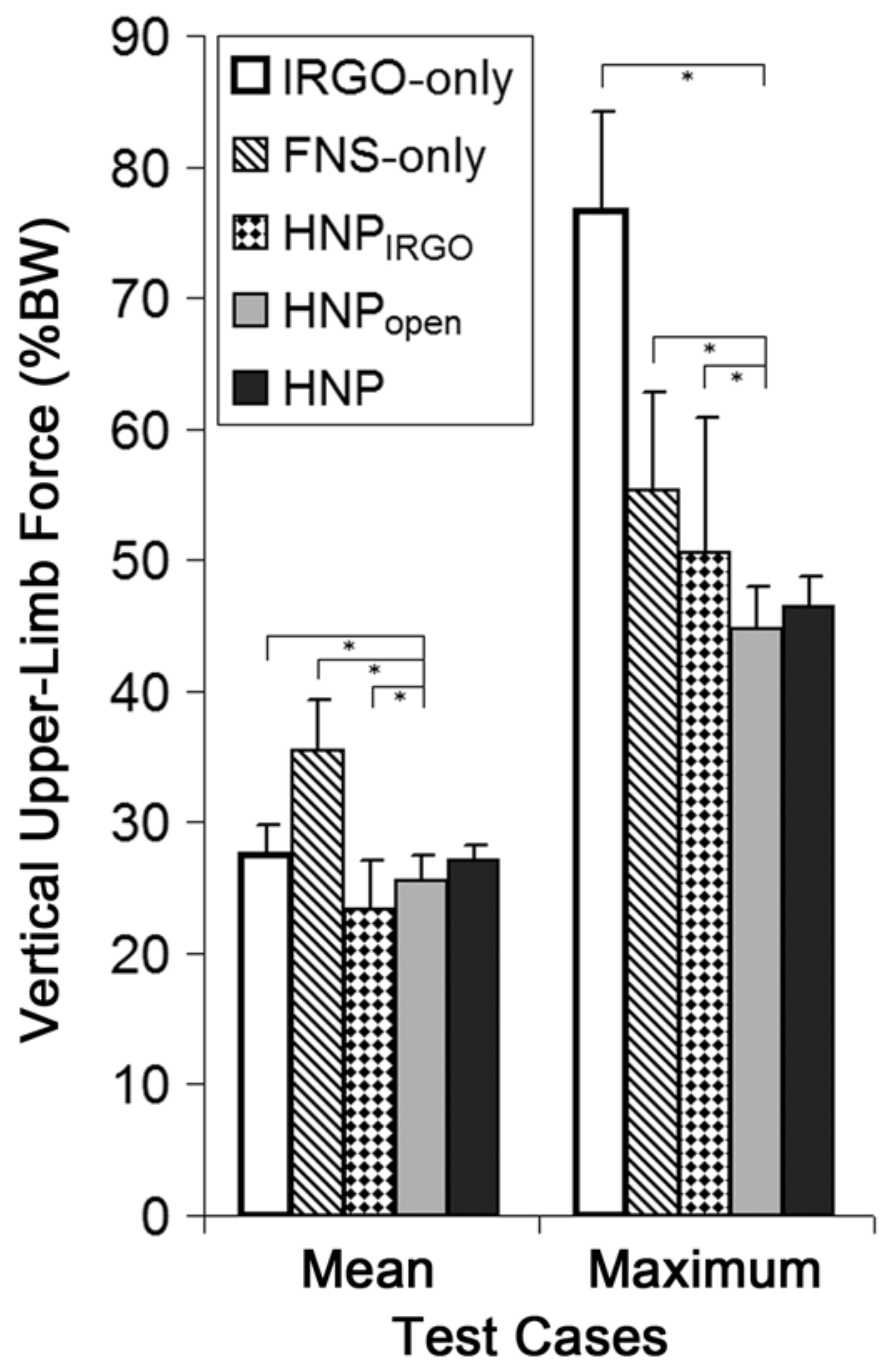

Figure 7.

Mean and average maximum vertical upper-limb forces (+1 standard deviation) in percentage body weight (\%BW) for all test cases. Masses of exoskeletons were not incorporated into normalization of applied forces. "Statistical significances between system configurations. FNS = functional neuromuscular stimulation, HNP = hybrid neuroprosthesis, IRGO = isocentric recriprocating gait orthosis. 
a 1:1 hip reciprocator. These reductions in upper-limb forces were apparent even with the weight of the exoskeleton, indicating that the exoskeleton was self-supporting.

\section{Variable-Constraint Hip Mechanism Effect on Hip Flexion and Step Length}

Figure 8 shows that the $\mathrm{HNP}_{\text {open }}$ (solid line) was able to achieve hip flexion that was from 60 to 115 percent greater when using VCHM as compared with $\mathrm{HNP}_{\text {IRGO }}$ (dashed line) with a fixed 1:1 hip coupling ratio $(p<0.001)$ and 53 percent (right: $p<0.001)$ to 58 percent (left: $p<0.001$ ) less than those exhibited during FNS-only walking (dash-dotted line). This suggests that the VCHM provided a degree of reciprocal coupling intermediate to the continuous $1: 1$ ratio of the IRGO and the uncoupled FNS only, which usually contains exaggerated movements during short walks [30].

The average step length of the left leg of the $\mathrm{HNP}_{\text {open }}$ was significantly longer than walking with the IRGO only $(p<0.001)$ or with the HNP IRGO $_{(}(p<0.001)$ (Table 2). Thus, gait speed with the $\mathrm{HNP}_{\text {open }}$ was faster than the IRGO only $(p<0.001)$ or the $\mathrm{HNP}_{\mathrm{IRGO}}(p<0.001)$. The step length between walking with the $\mathrm{HNP}_{\text {open }}$ and FNS only was not statistically different (left: $p=0.77$, right: $p=$
0.47) even though the hip and knee range of motion for FNS only was found to be significantly greater than with the $\mathrm{HNP}_{\text {open }}$. Figure 8 shows that the hip flexion during swing peaks at an average of $70^{\circ}$ and $30^{\circ}$ with FNS only and $\mathrm{HNP}_{\text {open }}$, respectively, while the hip flexion at the heel strike is essentially the same. Thus, the hip kinematics of the $\mathrm{HNP}_{\text {open }}$ for making a step was more efficient than FNS only at comparable step lengths. The speed $(p<0.001)$ and cadence (left: $p<0.001$, right: $p<0.001$ ) of the $\mathrm{HNP}_{\text {open }}$ is less than with FNS-only walking. This can be attributed to the participant's preference for automatically triggered (free cycling) stepping with FNS-only walking versus manually triggered stepping with $\mathrm{HNP}_{\text {open }}$ in addition to using plantar flexors for push-off during FNS-only walking.

\section{Functional Neuromuscular Stimulation Controller Effect on Hip Dynamics}

Figure 9 shows the average hip angles for the HNP (solid line) and $\mathrm{HNP}_{\text {open }}$ (dashed line) and the percentage of samples that the hip extensor stimulus was deactivated and stimulus frequency was increased when using the FNS controller. For the HNP, hip extensor stimulation was deactivated between 40 and 60 percent of the gait cycle at the end of the stance phase when the hip was
Left Leg
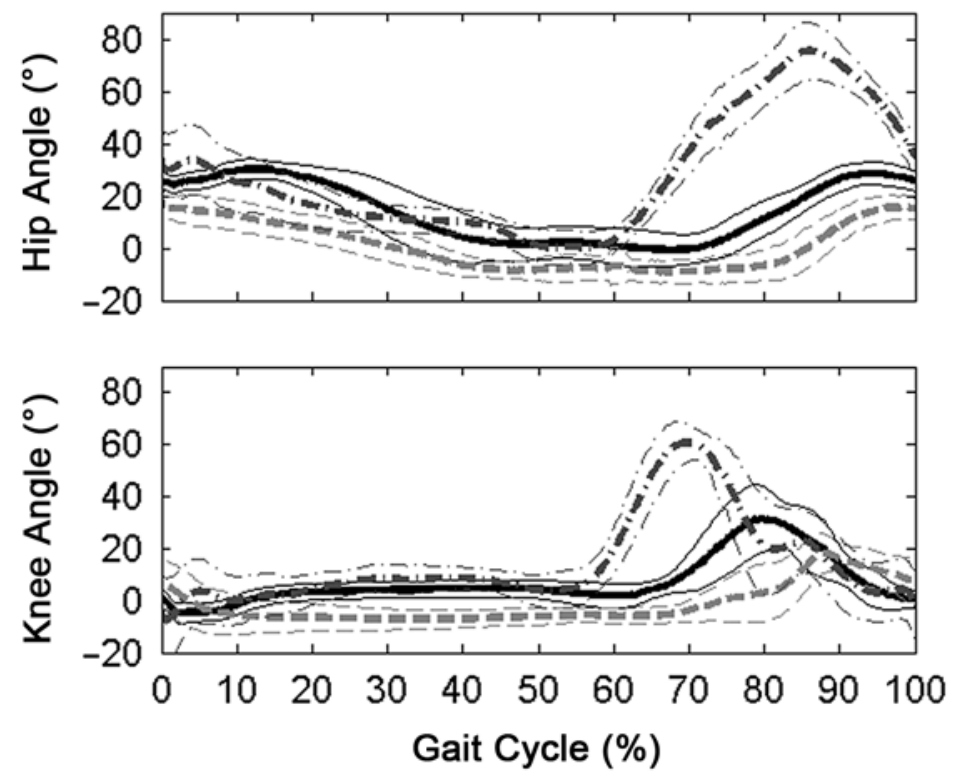

Right Leg
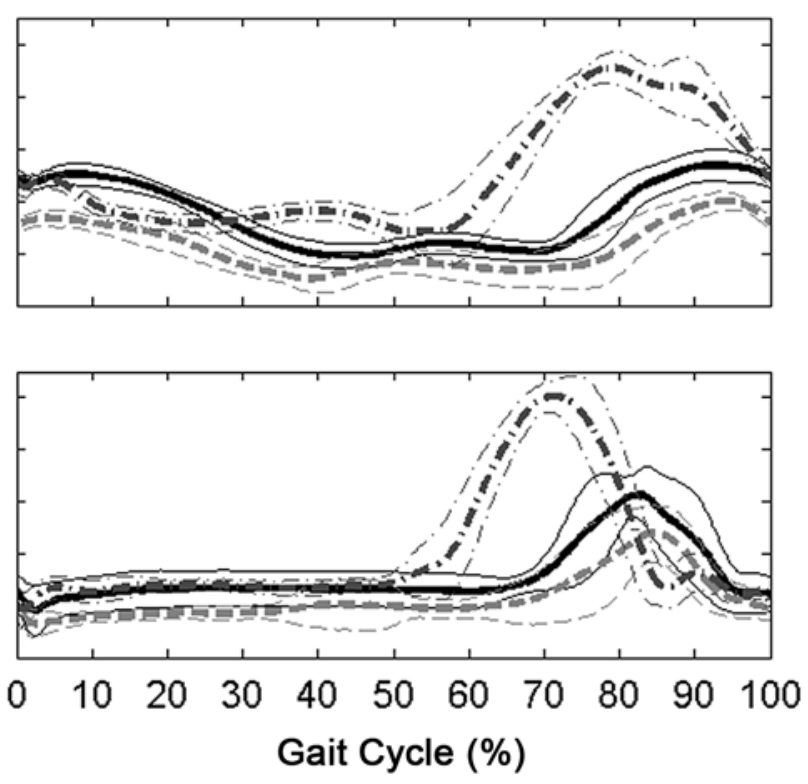

Figure 8.

Average hip and knee angles ( \pm 1 standard deviation) with respect to percentage gait cycle for walking with functional neuromuscular stimulation only (dashed-dotted line), hybrid neuroprosthesis (HNP)-isocentric reciprocating gait orthosis (dashed line), and HNP ${ }_{\text {open }}$ (solid line). 
Table 2.

Gait parameters measured with different device configurations.

\begin{tabular}{llccccc}
\hline \multirow{2}{*}{ Gait Parameters } & \multirow{2}{*}{ Side } & \multicolumn{4}{c}{ Device Configuration* (mean \pm SD) } \\
\cline { 3 - 7 } & & IRGO Only & FNS Only & HNP $_{\text {IRGO }}$ & HNP $_{\text {open }}$ & HNP \\
\hline Speed (m/s) & - & $0.12 \pm 0.24^{\mathrm{d}}$ & $0.43 \pm 0.27^{\mathrm{f}}$ & $0.13 \pm 0.19^{\mathrm{e}}$ & $0.25 \pm 0.16^{\mathrm{defh}}$ & $0.29 \pm 0.16^{\mathrm{h}}$ \\
Cadence (steps/min) & Left & $31 \pm 17$ & $60 \pm 3^{\mathrm{g}}$ & $32 \pm 11$ & $36 \pm 8^{\mathrm{g}}$ & $41 \pm 4$ \\
& Right & $36 \pm 9$ & $60 \pm 5$ & $37 \pm 6$ & $42 \pm 6$ & $42 \pm 4$ \\
Step Length (m) & Left & $0.24 \pm 0.10^{\mathrm{b}}$ & $0.41 \pm 0.07$ & $0.24 \pm 0.09^{\mathrm{c}}$ & $0.39 \pm 0.04^{\mathrm{bc}}$ & $0.39 \pm 0.06$ \\
& Right & $0.31 \pm 0.12$ & $0.42 \pm 0.12$ & $0.34 \pm 0.09$ & $0.35 \pm 0.14^{\mathrm{a}}$ & $0.44 \pm 0.08^{\mathrm{a}}$ \\
\hline
\end{tabular}

*Letters indicate statistically significant difference in measured parameter between different configurations.

${ }^{a}$ Statistically significant difference in right step length between $\mathrm{HNP}_{\text {open }}$ and HNP.

${ }^{\mathrm{b}}$ Statistically significant difference in left step length between $\mathrm{IRGO}_{\text {only }}$ and $\mathrm{HNP}_{\text {open }}$.

'Statistically significant difference in left step length between $\mathrm{HNP}_{\text {IRGO }}$ and $\mathrm{HNP}_{\text {open }}$.

${ }^{\mathrm{d}}$ Statistically significant difference in speed between $\mathrm{IRGO}_{\text {only }}$ and $\mathrm{HNP}_{\text {open }}$.

${ }^{\mathrm{e}}$ Statistically significant difference in speed between $\mathrm{HNP}_{\mathrm{IRGO}}$ and $\mathrm{HNP}_{\text {open }}$.

${ }^{\mathrm{f}}$ Statistically significant difference in speed between $\mathrm{FNS}_{\text {only }}$ and $\mathrm{HNP}_{\text {open }}$.

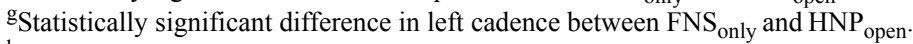

${ }^{\mathrm{h}}$ Statistically significant difference in speed between $\mathrm{HNP}_{\text {open }}$ and HNP.

$\mathrm{FNS}=$ functional neuromuscular stimulation, $\mathrm{HNP}=$ hybrid neuroprosthesis, $\mathrm{IRGO}=$ isocentric reciprocating gait orthosis, $\mathrm{SD}=\mathrm{standard}$ deviation.

fully extended for both sides. The percentage of samples that the FNS controller deactivated the hip extensor stimulation was on average 0.8 and 1.1 percent of the total walking duration for the left and right limbs, respectively. Increases of the stimulation frequency from 16.67 to $33.33 \mathrm{~Hz}$ occurred during stance for both hips. Frequency modulation occurred more often on the right side. This was because baseline stimulus frequency for the right gluteus maximus was set at a constant $16.67 \mathrm{~Hz}$, allowing for modulation to take place (Figure 3). The baseline stimulus frequencies for other hip extensors such as the adductor magnus, hamstrings, and left gluteus maximus were already set for the upper limit of $33.33 \mathrm{~Hz}$ for a portion of the stance phase. Similarly, no intermediate stimulus PW modulation occurred because baseline stimulus PW for all hip extensors were already set to their maximum safe levels for intramuscular electrodes [22]. Thus, the only PW modulation occurred when PW was reduced to zero when stimulation was deactivated.

Hip locking against flexion occurred infrequently throughout stance for both cases occurring 0.6 percent (left) and 1.5 percent (right) of the total walking duration with the FNS controller and 1.0 percent (left) and 0.1 percent (right) without it. When using the FNS controller, deactivation of hip extensor stimulation was generally coincident with hip locking such that support of the hips against flexion was transferred from FNS to the VCHM. Instances of hip uncoupling were also infrequent, occurring approximately 5.7 percent of the time when using the
FNS controller and 2.5 percent of the stride duration without it. Hip uncoupling during swing reduced the resistance from the contralateral limb and passive resistance of the VCHM [19], thus allowing for uninhibited motion. The average resistive torques applied by the VCHM at the hip with the FNS controller were 28 to 37 percent smaller than those with preprogrammed stimulation patterns (left: $p=0.02$, right: $p=0.05$ ) (Figure 10). The average maximum resistive torque of $10 \mathrm{Nm}$ for the HNP with the FNS controller was 17 percent lower on the left side $(p=0.03)$ than for $\mathrm{HNP}_{\text {open }}$ without FNS controller. Since there were more instances of hip uncoupling when using the FNS controller, the VCHM resisted the user less than with preprogrammed stimulation.

These results contributed to an 8 percent increase in the average hip range of motion when using the VCHM with the FNS controller relative to the VCHM with preprogrammed stimulation (left: $p=0.05$, right: $p=0.04$ ). This translated functionally into an increase in step length (Table 2) of the right side $(p=0.05)$, attributed primarily to increased left hip extension $(p=0.01)$. The increased step length facilitated a slight increase in gait speed averaged per stride of the HNP $(p=0.03)$.

\section{DISCUSSION}

The purpose of the HNP is to improve walking in individuals with paraplegia and minimize the upper-limb 
Left Leg

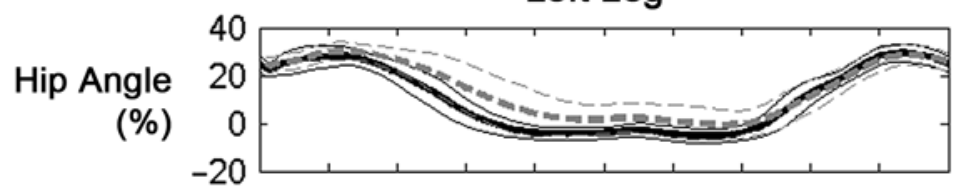

Stimulus 100

Deactivation 50

(\%)

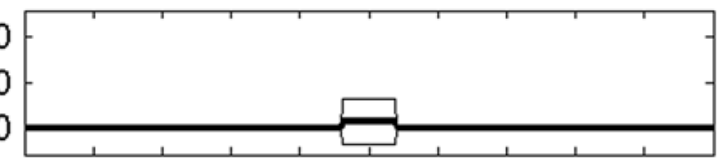

Stimulus

Frequency

Increase

(\%)

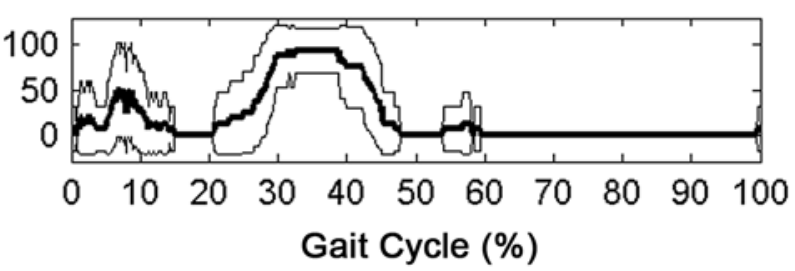

Right Leg
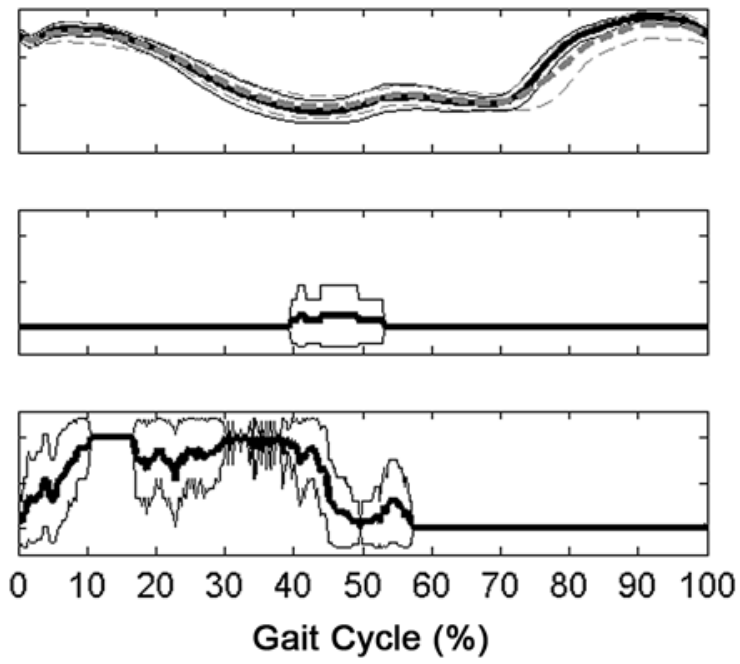

Figure 9.

Average hip angle, percentage of samples of hip extensor stimulus deactivation, and frequency increase from 16.67 to $33.33 \mathrm{~Hz}$ ( \pm 1 standard deviation) with respect to percentage gait cycle for hybrid neuroprosthesis (HNP) (solid line) and HNP open (dashed line).

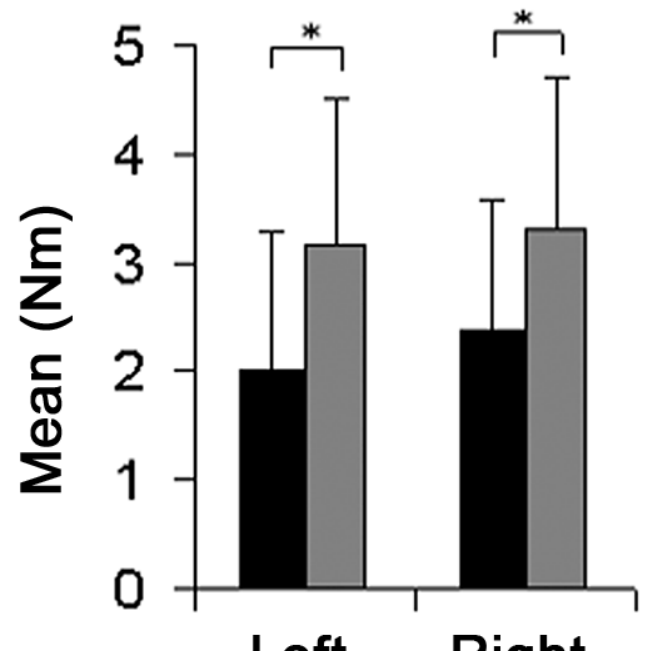

Left Right

Leg

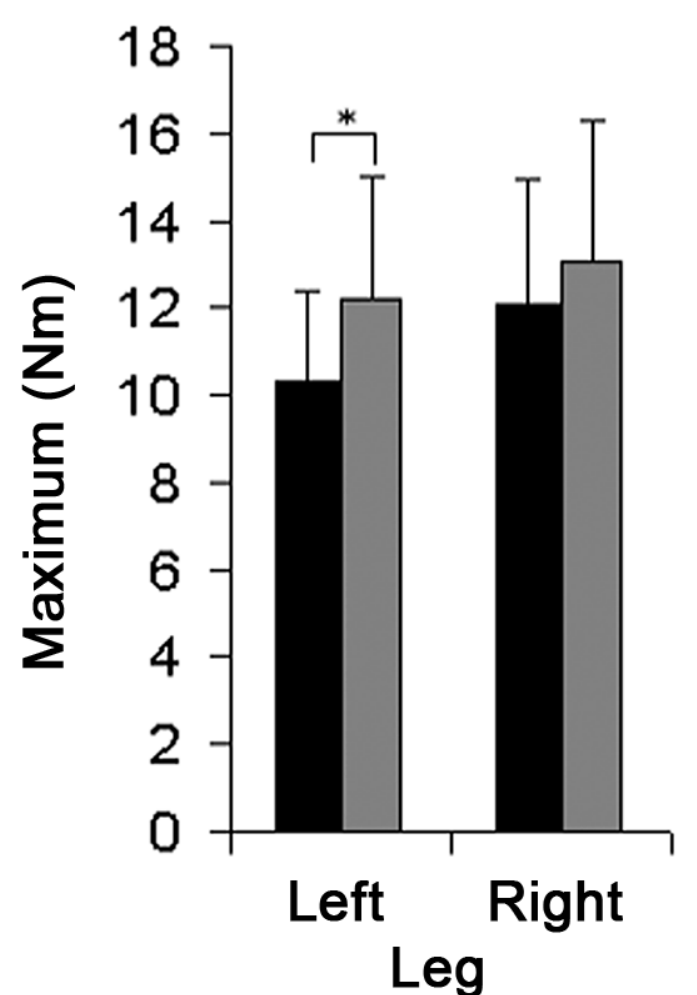

controller $\square$ baseline

Figure 10.

Mean and average maximum (+1 standard deviation) calculated resistive torque provided by variable-constraint hip mechanism during

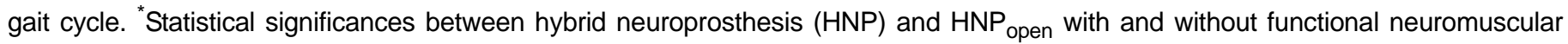
stimulation controller, respectively. 
forces for support. The HNP design explored in this study combined an exoskeleton (for support) with FNS (to provide power for movement). The exoskeleton with controllable joint constraints at the hips (VCHM) and knees (SCKM) provided support in combination with a rulebased feedback FNS controller that regulated hip motion as necessary. The cooperative interaction of the VCHM and the FNS controller can be summarized as follows. The VCHM prevented anterior trunk tilt as indicated by the measured flexion torque resisted by the VCHM. Hip uncoupling was more prevalent with the FNS controller (i.e., HNP) as evidenced by increases in stimulus frequency to the hip extensors during stance, which acted to drive the hips further into extension in coordination with VCHM. The absence of bilateral hip flexion caused the VCHM to uncouple the hips, thus allowing for unimpeded hip motion and modulation of stride length and velocity. This can be concluded from the fact that the flexion torque resisted by the VCHM when using the rule-based feedback FNS controller was significantly less than with preprogrammed open-loop stimulation. When full stance hip extension was achieved, the FNS controller responded by deactivating the hip extensors, transferring trunk and hip support to the VCHM, which was appropriately locked against flexion. The increased hip range of motion with the HNP led to functional improvements in step length and gait speed over the same exoskeleton under open-loop control (i.e., $\mathrm{HNP}_{\text {open }}$ ). There was a significant reduction in walking speed when comparing HNP with FNS-only walking and the speed improved when constraints of reciprocal coupling of the hips were removed. Similarly, even though the weight of the HNP was more than twice the weight of IRGO, the walking speed was considerably faster with HNP than with IRGO with reciprocal coupling. Thus, reducing the weight of the HNP and minimizing constraints of coupling has the potential to significantly improve the speed of walking.

The results in this study support the feasibility of this system in the following ways. First, the prototype VCHM was able to support the user, reducing the amount of anterior trunk tilt compared with FNS-only walking as well as the amount of upper-limb support relative to both IRGOonly and FNS-only walking. Noteworthy, the user upperlimb loading was reduced even though the exoskeleton component of the HNP was approximately one-third of the user's body weight, further indicating that the exoskeleton was self-supporting. Second, the rule-based modulation of the VCHM allowed for improved hip flexion relative to walking with an IRGO that had a fixed 1:1 hip coupling ratio, resulting in faster gait speeds. Increase in the hip flexion during swing with increasingly relaxed hip constraints (FNS only $>$ VCHM $>$ IRGO) generally resulted in an apparent increase in knee flexion (Figure 8). Since identical baseline stimulus parameters were implemented for all cases, this suggests that the amount of knee flexion is largely attributed to the thigh orientation and knee moment imposed by gravity on the lower leg. Third, the regulation of hip motion with rule-based feedback control of VCHM was shown to allow for step lengths comparable with FNS-only walking. Furthermore, the rule-based feedback control of FNS allowed for real-time increases in stimulation frequency to the hip extensors, leading to increased hip extension, reduced dependency of the VCHM to support against bilateral hip flexion, and increased step length and gait speed. Collectively, the results of this study demonstrated that the VCHM of the HNP with passive resistance of less than $4 \mathrm{Nm}$ and the potential to provide up to $42 \mathrm{Nm}$ of resistive torque [1920] was able to provide resistive moment of $10 \mathrm{Nm}$ for functional support to the user with reduced arm support and without compromising the stepping kinematics facilitated by the FNS as shown by step length comparable with unconstrained FNS-only walking.

Both the relative reduction in the upper-limb loads with the VCHM compared with FNS-only gait and the measured flexion torque resisted by the VCHM indicate that the VCHM effectively restricted against anterior trunk movements. However, the inability of the VCHM to maintain posture comparable with the IRGO only or $\mathrm{HNP}_{\text {IRGO }}$ may still contribute to upper-limb effort. The mean upperlimb force of the VCHM-based HNPs was significantly higher than that of the $\mathrm{HNP}_{\text {IRGO }}$. Conversely, the maximum upper-limb loads exhibited during use of VCHMbased HNPs were less than $\mathrm{HNP}_{\text {IRGO}}$, suggesting that the constraints of the fixed 1:1 hip coupling ratio of the IRGO may also negatively affect user effort.

When walking with the VCHM, the sagittal posture of the trunk depends on whether the hip can reach full extension at the end of stance. With maximal stimulation and the help of reciprocal hip coupling, full hip extension was rarely achieved as indicated by minimal instances in which the hip extensors were deactivated during walking with the HNP. Furthermore, the use of hip coupling during single stance to assist hip extension impeded contralateral hip flexion. This may be the primary reason that the hip flexion achieved with the VCHM-based HNPs 
was significantly smaller than that observed during FNSonly gait.

Depending on the muscle, the number of pulses per stride and total charge injected to the target hip extensors with the FNS controller were 20 to 50 percent larger than baseline levels (Figure 3). This was because the dominant action of the controller was to increase stimulus frequency during stance rather than deactivate muscles below baseline levels. Although injected charge was well within prescribed safety limits [22], the observed increases in stimulus frequency may expedite the onset of muscle fatigue since the concurrent reduction in stimulus pulse duration occurred relatively infrequently and decreased the average duty cycle of all the target hip extensors by only 2.2 percent. As muscles fatigue and end point cannot be reached, more effort is required by the user to maintain posture. On the other hand, if subject has strong stimulated hip extensors and end points are reached quickly, the stimulation duty cycle can be reduced and delay muscle fatigue. In this study, only stimulation to hip extensors was modulated by the rule-based feedback controller to maintain posture and power for forward progression to minimize user effort. By improving the FNS controller where stimulation PW and frequency are modulated based on an error from desired trajectory rather than from a baseline stimulation pattern used in FNS-only walking, the duty cycle may be reduced to delay muscle fatigue. In addition, implementation of plantar flexion control for push-off should further improve forward progression and reduce demand on hip extensors.

Operation of the HNP focused on achieving full hip extension at the end of stance. Full extension of the stance hip is crucial for maintaining forward propulsion and upright trunk posture. Future work to improve the postural control of this system should focus on increasing the mechanical efficiency of the VCHM [19] by reducing system compliance to provide more efficient reciprocal coupling and make the VCHM more resistant to bilateral hip flexion. In addition, hip coupling alone may not be sufficient in assisting hip extension without an active component. Thus, the incorporation of an active component to the exoskeleton may be necessary to augment hip extension and to reduce the high-stimulus parameter levels that may contribute to muscle fatigue. Since hip flexion is critical to providing walking on uneven terrain encountered in activities of daily living, an active component for hip flexion can ensure sufficient clearance during swing while assisting the hip extension of the stance leg as needed through reciprocal coupling. Once the stance hip is extended to neutral, the hip can be locked and decoupled to allow the swing leg to flex freely for increased step height and length. By improving hip extension, it is reasonable to expect that deactivation of stimulation to the hip extensors via the FNS controller will be more prevalent. This may lead to significant reductions in hip extensor duty cycle that would be beneficial in minimizing fatigue.

While assistive power is likely to increase the weight of the exoskeleton, there are other means of keeping the weight down. For example, we achieved a 30 percent reduction in weight by redesigning the rack and pinion of the original prototype used in this study and using carbon fiber for the lateral uprights. This modification brought the weight below that of commercially available motorized exoskeletons where most of the weight is distributed distally. Additional weight reduction will come from custom-designed hydraulic cylinders and valves. Our plan for assistive power is to use pressurized fluid located proximally on the corset and distributed distally to the hip and knee as needed. Thus, minimal additional weight will be added distally to minimize torque requirement for leg movements with FNS.

\section{CONCLUSIONS}

A sensor-based hip control of the HNP with exoskeletal VCHM and implanted FNS enabled an individual with paraplegia from SCI to maintain a more erect posture than with stimulated hip and trunk muscles assisted by the upper limbs when walking with FNS only and not as erect as when walking with IRGO. The upper-limb forces were significantly reduced with VCHM as compared with walking with IRGO or FNS only even with the weight of the exoskeleton. The speed of walking with variable hip coupling increased over continuous reciprocal hip coupling by increasing step length and was comparable to FNS-only walking with unrestrained hip motion. When the FNS controller of hip muscles was active in addition to controller of the VCHM, the uncoupling of hips with the VCHM occurred more often and resistive moments provided with bracing for stability were reduced. 


\section{ACKNOWLEDGMENTS}

\section{Author Contributions:}

Study concept and design: C. S. To, R. Kobetic, R. J. Triolo. Acquisition of data: C. S. To, T. C. Bulea, M. L. Audu, R. Kobetic. Analysis and interpretation of data: C. S. To, R. Kobetic, T. C. Bulea, R. J. Triolo.

Drafting of manuscript: C. S. To, R. Kobetic.

Statistical analysis: C. S. To.

Obtained funding: R. Kobetic, R. J. Triolo.

Administrative, technical, or material support: R. Kobetic, T. C. Bulea, J. R. Schnellenberger, G. Pinault, M. L. Audu, R. J. Triolo. Study supervision: R. Kobetic, R. J. Triolo.

Financial Disclosure: The authors have declared that no competing interests exist.

Funding/Support: This material was based on work supported in part by the Department of Veterans Affairs Rehabilitation Research and Development Service (grants B3463R and A6404R) and the Department of Defense Congressionally Directed Medical Research Program (grant PR043074).

Additional Contributions: We thank Drs. Robert F. Kirsch, Patrick E. Crago, and Roger D. Quinn for their advice and contribution. Dr. To is now at Ford Corporation, Auburn Hills, Michigan. Dr. Bulea is now with the Functional and Applied Biomechanics Section at the National Institutes of Health, Bethesda, Maryland.

Institutional Review: The participant signed a consent form approved by the institutional review board of the Louis Stokes Cleveland Department of Veterans Affairs Medical Center.

Participant Follow-Up: The authors plan to inform the participant of the publication of this study.

\section{REFERENCES}

1. Rose GK. The principles and practice of hip guidance articulations. Prosthet Orthot Int. 1979;3(1):37-43.

[PMID:471704]

2. Major RE, Stallard J, Rose GK. The dynamics of walking using the hip guidance orthosis (hgo) with crutches. Prosthet Orthot Int. 1981;5(1):19-22. [PMID:7279608]

3. Douglas R, Larson PF, D'Ambrosia R, McCall RE. The LSU reciprocating gait orthosis. Orthopedics. 1983;6(7): 834-39.

4. Marsolais EB, Kobetic R, Polando G, Ferguson K, Tashman S, Gaudio R, Nandurkar S, Lehneis HR. The Case Western Reserve University hybrid gait orthosis. J Spinal Cord Med. 2000;23(2):100-108. [PMID:10914350]

5. Dall PM, Müller B, Stallard I, Edwards J, Granat MH. The functional use of the reciprocal hip mechanism during gait for paraplegic patients walking in the Louisiana State University reciprocating gait orthosis. Prosthet Orthot Int. 1999;23(2):152-62. [PMID:10493143]

6. IJzerman MJ, Baardman G, Hermens HJ, Veltink PH, Boom HB, Zilvold G. The influence of the reciprocal cable linkage in the advanced reciprocating gait orthosis on paraplegic gait performance. Prosthet Orthot Int. 1997;21(1): 52-61. [PMID:9141126]

7. Hirokawa SM, Grimm M, Le T, Solomonow M, Baratta RV, Shoji H, D'Ambrosia RD. Energy consumption in paraplegic ambulation using the reciprocating gait orthosis and electrical stimulation of the thigh muscles. Arch Phys Med Rehabil. 1990;71(9):687-94. [PMID:2375676]

8. Jefferson RJ, Whittle MW. Performance of three walking orthoses for the paralysed: A case study using gait analysis. Prosthet Orthot Int. 1990;14(3):103-10. [PMID:2095527]

9. Motloch WM. Principles of orthotic management for child and adult paraplegia and clinical experience with the isocentric RGO. Proceedings of the 7th World Congress of the International Society for Prosthetics and Orthotics; 1992 Jun 28-Jul 3; Chicago, IL. p. 28.

10. Pedotti A, Ferrarin M, Quintern J, Riener R. Neuroprosthetics: From basic research to clinical application. Berlin (Germany): Springer; 1996. p. 493-502.

11. Ohta Y, Yano H, Suzuki R, Yoshida M, Kawashima N, Nakazawa K. A two-degree-of-freedom motor-powered gait orthosis for spinal cord injury patients. Proc Inst Mech Eng H. 2007;221(6):629-39. [PMID:17937202] http://dx.doi.org/10.1243/09544119JEIM55

12. Nouri BM, Zaidan A. Computer control of a powered two degree freedom reciprocating gait orthosis. ISA Trans. 2006;45(2):249-58. [PMID:16649569] http://dx.doi.org/10.1016/S0019-0578(07)60193-1

13. Arazpour M, Chitsazan A, Hutchins SW, Ghomshe FT, Mousavi ME, Takamjani EE, Aminian G, Rahgozar M, Bani MA. Evaluation of a novel powered hip orthosis for walking by a spinal cord injury patient: A single case study. Prosthet Orthot Int. 2012;36(1):105-12.

[PMID:22235110] http://dx.doi.org/10.1177/0309364611431482

14. Kim G, Kang S, Kang S, Ryu J, Mun M, Kim K. Unlockable knee joint mechanism for powered gait orthosis. Int $\mathrm{J}$ Prec Eng Manufactur. 2009;10(3):83-89. http://dx.doi.org/10.1007/s12541-009-0051-y

15. Arazpour M, Chitsazan A, Hutchins SW, Mousavi ME, Takamjani EE, Ghomshe FT, Aminian G, Rahgozar M, Bani MA. Evaluation of a novel powered gait orthosis for walking by a spinal cord injury patient. Prosthet Orthot Int. 2012;36(2):239-46. [PMID:22368113] http://dx.doi.org/10.1177/0309364611435690

16. Arazpour M, Bani MA, Hutchins SW. Reciprocal gait orthoses and powered gait orthoses for walking by spinal cord injury patients. Prosthet Orthot Int. 2013;37(1):14-21. [PMID:22588849] http://dx.doi.org/10.1177/0309364612444665

17. del-Ama AJ, Koutsou AD, Moreno JC, de-los-Reyes A, Gil-Agudo A, Pons JL. Review of hybrid exoskeletons to 
restore gait following spinal cord injury. J Rehabil Res Dev. 2012;49(4):497-514. [PMID:22773254]

http://dx.doi.org/10.1682/JRRD.2011.03.0043

18. Yang L, Granat MH, Paul JP, Condie DN, Rowley DI. Further development of hybrid functional electrical stimulation orthoses. Spinal Cord. 1996;34(10):611-14.

[PMID:8896128] http://dx.doi.org/10.1038/sc.1996.109

19. To CS, Kobetic R, Schnellenberger JR, Audu ML, Triolo RJ. Design of a variable constraint hip mechanism for a hybrid neuroprosthesis to restore gait after spinal cord injury. IEEE/ ASME Trans Mechatronics. 2008;13(2):197-205.

20. To CS. Closed-loop control and variable constraint mechanisms of a hybrid neuroprosthesis to restore gait after spinal cord injury [PhD dissertation]. [Cleveland]: Department of Biomedical Engineering, Case Western Reserve University; 2010.

21. Kobetic R, To CS, Schnellenberger JR, Audu ML, Bulea TC, Gaudio R, Pinault G, Tashman S, Triolo RJ. Development of hybrid orthosis for standing, walking, and stair climbing after spinal cord injury. J Rehabil Res Dev. 2009; 46(3):447-62. [PMID:19675995] http://dx.doi.org/10.1682/JRRD.2008.07.0087

22. Mortimer JT, Kaufman D, Roessman U. Intramuscular electrical stimulation: Tissue damage. Ann Biomed Eng. 1980;8(3):235-44. [PMID:7224246] http://dx.doi.org/10.1007/BF02364479

23. Crago PE, Peckham PH, Thrope GB. Modulation of muscle force by recruitment during intramuscular stimulation. IEEE Trans Biomed Eng. 1980;27(12):679-84. [PMID:6970162] http://dx.doi.org/10.1109/TBME.1980.326592

24. Crago PE, Mortimer JT, Peckham PH. Closed-loop control of force during electrical stimulation of muscle. IEEE Trans Biomed Eng. 1980;27(6):306-12. [PMID:7390527]

25. Carroll SG, Triolo RJ, Chizeck HJ, Kobetic R, Marsolais EB. Tetanic responses of electrically stimulated paralyzed muscle at varying interpulse intervals. IEEE Trans Biomed Eng. 1989;36(7):644-53. [PMID:2787276] http://dx.doi.org/10.1109/10.32096

26. To CS, Kobetic R, Bulea TC, Audu ML, Schnellenberger JR, Pinault G, Triolo RJ. Stance control knee mechanism for lower-limb support in hybrid neuroprosthesis. J Rehabil Res Dev. 2011;48(7):839-50. [PMID:21938668] http://dx.doi.org/10.1682/JRRD.2010.07.0135

27. To CS, Kobetic R, Bulea TC, Audu ML, Schnellenberger JR, Pinault G, Triolo RJ. Sensor- based stance control with orthosis and functional neuromuscular stimulation for walking after spinal cord injury. J Prosthet Orthot. 2012;24(3): 124-32. http://dx.doi.org/10.1097/JPO.0b013e3182627a13

28. Trier S, Vrabec T, Weisgarber J. Using functional electrical stimulation to restore movement to individuals with neuromuscular disabilities. Natick (MA): MATLAB Digest, Academic Edition; 2008.

29. Agarwal S, Kobetic R, Nandurkar S, Marsolais EB. Functional electrical stimulation for walking in paraplegia: 17year follow-up of 2 cases. J Spinal Cord Med. 2003;26(1): 86-91. [PMID:12830975]

30. Kobetic R, Marsolais EB. Synthesis of paraplegic gait with multichannel functional neuromuscular stimulation. IEEE Trans Rehabil Eng. 1994;2(2):66-79. http://dx.doi.org/10.1109/86.313148

31. Audu ML, To CS, Kobetic R, Triolo RJ. Gait evaluation of a novel hip constraint orthosis with implication for walking in paraplegia. IEEE Trans Neural Syst Rehabil Eng. 2010; 18(6):610-18. [PMID:20378478] http://dx.doi.org/10.1109/TNSRE.2010.2047594

Submitted for publication October 31, 2012. Accepted in revised form September 17, 2013.

This article and any supplementary material should be cited as follows:

To CS, Kobetic R, Bulea TC, Audu ML, Schnellenberger JR, Pinault G, Triolo RJ. Sensor-based hip control with hybrid neuroprosthesis for walking in paraplegia. J Rehabil Res Dev. 2014;51(2):229-44. http://dx.doi.org/10.1682/JRRD.2012.10.0190

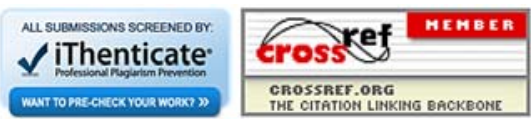

